\title{
Perspectivas futuras sobre participação social na ótica de adolescentes com paralisia cerebral e suas mães
}

\section{Future perspectives on the social participation of adolescents with cerebral palsy and their mothers}

\author{
Priscila Bianchi Lopes $^{1}$, Thelma Simões Matsukura ${ }^{2}$
}

http://dx.doi.org/10.11606/issn.2238-6149.v29i1p19-26

Bianchi-Lopes P, Matsukura TS. Perspectivas futuras sobre participação social na ótica de adolescentes com paralisia cerebral e suas mães. Rev Ter Ocup Univ São Paulo. 2018 jan.-abr.;29(1):19-26.

RESUMO: Objetivo: Conhecer as perspectivas futuras em relação a participação social de adolescentes com Paralisia Cerebral, sob a ótica dos próprios adolescentes e de seus familiares. Método: Trata-se de um estudo exploratório de abordagem qualitativa. Participaram 7 adolescentes com PC, com idade entre 11 e 17 anos, e suas mães. Foram utilizados, para a coleta de dados, dois roteiros de entrevista semiestruturada, um formulário de identificação e o GMFCS Sistema de Classificação da Função Motora Grossa - Questionário do Relato Familiar. Os dados gerados a partir das entrevistas foram analisados através da metodologia do Discurso do Sujeito Coletivo. Resultados: Os principais resultados indicaram que os adolescentes desejam seguirem uma carreira profissional, continuarem estudando e estarem perto da família e de amigos. Em relação às mães, estas demonstraram preocupações com o surgimento de questões da adolescência, como o envolvimento com pares, namoros, questionamento das práticas parentais e autoafirmação e suas expectativas em relação ao futuro dos filhos como a independência financeira e autonomia. Conclusão: Compreende-se que a presente pesquisa fornece elementos para intervenções terapêuticas ocupacionais que visam a ampliação e o fortalecimento da participação social de adolescentes com PC.

DESCRITORES: Paralisia cerebral; Adolescentes; Participação social; Futuro; Família; Terapia ocupacional.
Bianchi-Lopes P, Matsukura TS. Future perspectives on the social participation of adolescents with cerebral palsy and their mothers. Rev Ter Ocup Univ São Paulo. 2018 Jan.-Apr.;29(1):19-26.

\begin{abstract}
Aim: To understand the future aspirations regarding the social participation of adolescents with $\mathrm{CP}$, from the perspective of the adolescents and their families. Method: This exploratory study used a qualitative methodology. The participants were seven adolescents with CP (aged from 11 to 17 years old) and their mothers. For collecting data, it was used two semi-structured interview script, a descriptive questionnaire and the GMFCS Gross Motor Function Classification System - Family Report Questionnaire. Data from interviews were analyzed through the methodology of the Collective Subject Discourse. Results: The main results indicated that the adolescent's wishes and hopes about their social participation in the future are related to professional career, to future studies and to a close relationship with family and friends. Mothers evidenced concerns about issues of the adolescence, such as involvement with peers, dating, challenging parental practices and self-affirmation, and their expectations regarding to the future of their children, such as financial independence and autonomy. Conclusion: It is expected that this study provides elements for occupational therapist's practices that aim at expanding and strengthening the social participation of adolescents with $\mathrm{CP}$.
\end{abstract}

KEYWORDS: Cerebral palsy; Adolescents; Social participation; Future; Family; Occupational therapy.

Este estudo está atrelado a pesquisa mais ampla de mestrado, desenvolvida no Programa de Pós Graduação em Terapia Ocupacional da UFSCar, intitulada de "Parentalidade, Participação e Suporte Social: dando voz aos adolescentes com paralisia cerebral e a suas mães", desenvolvida pelas autoras deste estudo.

1. Mestrado em Terapia Ocupacional; Universidade Federal de São Carlos, São Carlos, São Paulo, Brasil. ORCID: https://orcid.org/00000002-5272-5180. E-mail: pri.bianchi.to@gmail.com

2. Professora Doutora, Departamento de Terapia Ocupacional, Universidade Federal de São Carlos, São Carlos, São Pulo, Brasil. ORCID: https://orcid.org/0000-0003-3812-3893. E-mail: thelmamatsukura@gmail.com

Endereço para correspondência: Priscila B. Lopes. Universidade Federal de São Carlos. Rodovia Washington Luis, km 235. São Carlos, São Paulo, Brasil. CEP: 13565-905. E-mail: pri.bianchi.to@gmail.com 


\section{INTRODUÇÃO}

Paralisia Cerebral (PC) descreve um
grupo de desordens permanentes do
desenvolvimento do movimento e da postura que são atribuídas a distúrbios não progressivos ocorridos durante o desenvolvimento fetal ou no cérebro infantil ${ }^{1}$. Sabe-se que a PC constitui-se na causa mais comum de deficiência física na infância e tem prevalência de 2 a 2,5 por 1000 nascimentos no mundo ${ }^{1,2}$.

Dentre as dificuldades que crianças e adolescentes com PC experienciam destacam-se os problemas relacionados à mobilidade, resolução de problemas, socialização e comunicação associados a limitações em atividades. Além disso, esta população está em risco de ter prejudicada sua participação em atividades sociais e de lazer, as quais são cruciais para a promoção de amizades, para o desenvolvimento de interesses e também para gerar bem estar ${ }^{3}$.

Embora a participação em atividades sociais para adolescentes com deficiência e condições crônicas de saúde tenha ganhado um pouco mais de atenção nos últimos anos ${ }^{3,4}$, a literatura tem apontado uma lacuna no conhecimento em relação a intensidade e a forma como crianças e adolescentes com deficiências físicas tem a oportunidade de estar envolvidos e apreciarem atividades de lazer em casa e na comunidade, revelando que estas ainda são problemáticas emergentes de pesquisa ${ }^{5,6}$. Nesse sentido, destaca-se que a participação social é um fenômeno complexo que necessita ser estudado de forma mais aprofundada ${ }^{7}$ e ressalta-se aqui a importância de estudos que somem esforços em aprofundar o conhecimento sobre esta população $0^{3,8}$.

Além disso, reforça-se que embora a pesquisa sobre a adolescência e a transição para a vida adulta por jovens com PC tenha crescido nas últimas décadas ainda há muitos elementos não compreendidos em relação a sua participação social ${ }^{7,9}$. Não obstante, tem-se revelado que a participação social está associada com a promoção de saúde, comportamentos sociais e outros resultados positivos para adolescentes ${ }^{7}$.

Estudos recentes apontam que quando a família tende a tratar a criança e o adolescente com deficiência da mesma maneira que os demais membros da família eles podem sentir-se mais seguros, com maior participação social e melhor percepção de qualidade de vida ${ }^{10,11}$.

$\mathrm{Na}$ adolescência, a participação social prepara o adolescente para aspectos importantes da fase adulta como o trabalho, o casamento e o lazer. Além disso, a participação social permite que os adolescentes ampliem sua rede social com pessoas que não são do contexto do lar ou da rotina escolar, e essas experiências são importantes para o desenvolvimento de habilidades e para o cumprimento dos papéis de adultos ${ }^{12}$.

A literatura aponta que os adolescentes com PC, precisam ter a oportunidade de discutirem seus sentimentos, medos e expectativas em relação ao futuro, tendo em vista que eles estão em um processo contínuo de mudança na maneira como se enxergam e se definem ${ }^{13}$.

Cussen et al. ${ }^{13}$, através de uma pesquisa qualitativa realizada na Austrália, objetivaram compreender as expectativas e esperanças de adolescentes com PC para o futuro. Como resultados os adolescentes expressaram o desejo de estar perto da família, de amigos e de seus animais de estimação, de planejar a carreira profissional, de viver em casa e de forma independente e de fazer uma viagem em um momento futuro. Os pesquisadores reforçaram a relevância de se buscar informações a partir dos próprios adolescentes para que seja possível dar suporte às expectativas dos mesmos.

Nesse sentido, reforça-se que para entender os problemas e os desafios que adolescentes com deficiência encontram em seu caminho para a vida adulta, é necessário abordar as perspectivas dos próprios adolescentes com PC e de seus familiares.

Compreende-se também que o conhecimento neste campo pode ser utilizado por terapeutas ocupacionais e outros profissionais que trabalham com adolescentes de modo a ampliar e promover seu desenvolvimento psicossocial ${ }^{5}$.

\section{OBJETIVO}

Este estudo objetivou conhecer as perspectivas futuras em relação a participação social de adolescentes com PC, sob a ótica dos próprios adolescentes e de suas mães.

\section{MÉTODO}

Trata-se de um estudo exploratório, descritivo, transversal e de abordagem qualitativa. Este estudo está atrelado a pesquisa mais ampla de mestrado, desenvolvida no Programa de Pós-Graduação em Terapia Ocupacional da UFSCar, intitulada de "Parentalidade, participação e suporte social: dando voz aos adolescentes com paralisia cerebral e a suas mães", desenvolvida pelas autoras deste estudo.

\section{Participantes}

Foram participantes desta pesquisa 7 adolescentes com PC, com idade entre 11 e 17 anos e suas mães. 
Aamostra participante foi eleita de forma intencional e identificada com objetivo e por conveniência ${ }^{14}$. Como critérios para participação nesta pesquisa, preconizou-se que os familiares deveriam residir com o adolescente com PC há, pelo menos, 3 anos e os adolescentes deveriam ter diagnóstico de Paralisia Cerebral, conforme informado por técnicos da saúde ou educação.

\section{Local}

Este estudo foi realizado em uma cidade de médio porte, da região centro-oeste do estado de São Paulo, Brasil, com população aproximada de 241.389 habitantes ${ }^{15}$. As entrevistas foram realizadas nas casas dos participantes, exceto por dois participantes que preferiram realizar a entrevista na escola onde estudavam.

\section{Instrumentos}

Foi aplicado junto as mães um questionário para caracterizar o perfil socioeconômico e obter informações gerais como, por exemplo, a escolaridade, situação conjugal, número de filhos.

Foi utilizada também a versão traduzida do GMFCS Family Report Questionnaire ${ }^{16}$ para identificar o nível de função motora grossa dos participantes, considerando as restrições na mobilidade e assim caracterizá-los em termos de diferentes níveis de funcionalidade.

Para conhecer as perspectivas futuras em relação a participação social dos adolescentes com PC, foram elaborados dois roteiros de entrevista semi-estruturada. Os adolescentes responderam a quatro questões sobre seu futuro, projetos, sonhos e desafios. As mães dos adolescentes foram convidadas a responder questões sobre suas expectativas quanto ao futuro dos filhos e também sobre os desafios futuros como pais.

\section{Procedimentos}

Os adolescentes participantes foram indicados por um técnico de saúde do equipamento de reabilitação onde eram atendidos. Após a apresentação dos objetivos do estudo, foi feito o convite para participação da pesquisa aos adolescentes e aos seus responsáveis. Os adolescentes que aceitaram participar foram então entrevistados individualmente assim como seus responsáveis, sendo todos representados por mães.

\section{Procedimentos éticos}

Esse estudo foi submetido à análise do Comitê de Ética em Pesquisa em Seres Humanos da UFSCar e aprovado sob o parecer $\mathrm{n}^{\circ} 794.535$.

\section{Análise de dados}

Todas as entrevistas foram áudio-gravadas e a partir das gravações foi realizada a transcrição literal.

Os dados provenientes das entrevistas foram analisados qualitativamente através do método Discurso do Sujeito Coletivo (DSC). Esse método tem sido utilizado mundialmente em diversos estudos em diferentes áreas de conhecimento tais como saúde e educação. Fundamentado pela Teoria da Representação Social o DSC envolve a reunião, em discursos-sínteses, dos conteúdos e argumentos que conformam opiniões semelhantes, o que permite a representação da opinião de uma coletividade, através de um único discurso sem perder a natureza essencialmente discursiva e qualitativa da opinião. Deste modo, o DSC consiste em um conjunto de operações que culminam em depoimentos coletivos, os quais são constructos formados a partir de estratos literais do conteúdo mais significativo dos diversos depoimentos cujos sentidos são similares ${ }^{17,18}$.

\section{RESULTADOS}

Os dados socioeconômicos e informações gerais sobre o perfil dos participantes, assim como a classificação do GMFCS estão descritos nos Quadros 1 e 2 .

Como pode ser observado no Quadro 1, 4 participantes tinham idade entre 14 e 15 anos, e apenas 1 participante com idade de 17 anos.

Dos 7 adolescentes participantes 6 se encontram inseridos na rede regular de ensino e somente 1 frequenta apenas a escola especial.

Além disso, dentre os 7 participantes, 6 fazem acompanhamento especializado semanal em Fisioterapia e Terapia Ocupacional e apenas 1 não está realizando tratamento.

Com relação à função motora grossa, a classificação do GMFCS variou entre os níveis mais leves I e II, com 3 participantes, e os níveis mais graves IV e V, com 4 participantes.

O perfil das mães participantes da pesquisa está descrito no Quadro 2 
Quadro 1 - Perfil dos Adolescentes participantes do estudo

\begin{tabular}{|c|c|c|c|c|c|c|c|}
\hline & $\begin{array}{c}\text { Idade } \\
\text { em anos }\end{array}$ & Sexo & Escolaridade & Tipo de Escola & Percurso Educacional & $\begin{array}{l}\text { Nível de } \\
\text { Função } \\
\text { Motora* }\end{array}$ & $\begin{array}{c}\text { Realiza algum } \\
\text { tratamento }\end{array}$ \\
\hline A1 & 11 & Masculino & $\begin{array}{l}\text { Cursando o } 5^{\circ} \text { ano do } \\
\text { Ensino Fundamental }\end{array}$ & $\begin{array}{l}\text { Escola Municipal } \\
\text { Regular }\end{array}$ & $\begin{array}{l}\text { Sempre estudou em } \\
\text { escola regular }\end{array}$ & II & $\begin{array}{c}\text { Fisioterapia e } \\
\text { Terapia Ocupacional }\end{array}$ \\
\hline A2 & 17 & Masculino & $\begin{array}{l}\text { Cursando o } 8^{\circ} \text { ano do } \\
\text { Ensino Fundamental }\end{array}$ & $\begin{array}{l}\text { Escola Especial } \\
\text { Particular }\end{array}$ & $\begin{array}{c}\text { Estudou em escola } \\
\text { regular }\end{array}$ & IV & $\begin{array}{c}\text { Fisioterapia e } \\
\text { Terapia Ocupacional }\end{array}$ \\
\hline $\mathbf{A 3}$ & 11 & Feminino & $\begin{array}{l}\text { Cursando o } 5^{\circ} \text { ano do } \\
\text { Ensino Fundamental }\end{array}$ & $\begin{array}{c}\text { Escola Municipal } \\
\text { Regular }\end{array}$ & $\begin{array}{l}\text { Sempre estudou em } \\
\text { escola regular }\end{array}$ & IV & $\begin{array}{c}\text { Fisioterapia e } \\
\text { Terapia Ocupacional }\end{array}$ \\
\hline A4 & 15 & Feminino & $\begin{array}{l}\text { Cursando o } 8^{\circ} \text { ano do } \\
\text { Ensino Fundamental }\end{array}$ & $\begin{array}{l}\text { Escola Municipal } \\
\text { Regular e Escola } \\
\text { Particular Especial }\end{array}$ & $\begin{array}{l}\text { Sempre estudou em } \\
\text { escola regular e há } 2 \\
\text { anos frequenta também } \\
\text { a escola especial }\end{array}$ & IV & $\begin{array}{c}\text { Fisioterapia, } \\
\text { Hidroterapia e } \\
\text { Terapia Ocupacional }\end{array}$ \\
\hline A5 & 14 & Feminino & $\begin{array}{l}\text { Cursando o } 1^{\circ} \text { ano do } \\
\text { Ensino Médio }\end{array}$ & $\begin{array}{l}\text { Escola Estadual } \\
\text { Regular }\end{array}$ & $\begin{array}{l}\text { Sempre estudou em } \\
\text { escola regular }\end{array}$ & I & Fisioterapia \\
\hline A6 & 14 & Feminino & $\begin{array}{l}\text { Cursando o } 9^{\circ} \text { ano do } \\
\text { Ensino Fundamental }\end{array}$ & $\begin{array}{c}\text { Escola Particular } \\
\text { Regular }\end{array}$ & $\begin{array}{l}\text { Sempre estudou em } \\
\text { escola regular }\end{array}$ & II & Fisioterapia \\
\hline A7 & 15 & Feminino & $\begin{array}{l}\text { Cursando o } 8^{\circ} \text { ano do } \\
\text { Ensino Fundamental }\end{array}$ & $\begin{array}{l}\text { Escola Estadual } \\
\text { Regular }\end{array}$ & $\begin{array}{c}\text { Estudou durante } 4 \text { anos } \\
\text { em escola especial } \\
\text { e depois passou a } \\
\text { frequentar a escola } \\
\text { regular }\end{array}$ & V & \\
\hline
\end{tabular}

*Conforme o Sistema de Classificação de Função Motora Grossa para Paralisia Cerebral - GMFCS.

Quadro 2 - Perfil das Mães participantes do estudo

\begin{tabular}{|l|c|c|c|c|c|c|}
\hline & Idade em anos & Profissão & Escolaridade & $\begin{array}{c}\text { Situação } \\
\text { Conjugal }\end{array}$ & $\begin{array}{c}\text { Número } \\
\text { de Filhos }\end{array}$ & $\begin{array}{c}\text { Renda Familiar } \\
\text { em salários } \\
\text { mínimos* }\end{array}$ \\
\hline M1 & 29 & Dona de casa & Ensino Fundamental Incompleto & Casada & 3 & $1-2$ \\
\hline M2 & 54 & Dona de casa & Ensino Fundamental Incompleto & Viúva & 5 & $6-8$ \\
\hline M3 & 28 & Dona de casa & Ensino Fundamental Completo & Casada & 3 & $2-4$ \\
\hline M4 & 52 & Dona de casa & Ensino Médio Completo & Casada & 3 & $2-4$ \\
\hline M5 & 53 & Comerciante & Ensino Fundamental Completo & Casada & 4 & $4-6$ \\
\hline M6 & 47 & Administradora & Ensino Superior Completo & Casada & 2 & $4-6$ \\
\hline M7 & 38 & Dona de casa & Ensino Fundamental Incompleto & Divorciada & 3 & $1-2$ \\
\hline
\end{tabular}

Nota: *valor do salário mínimo vigente na época de coleta de dados $=\mathrm{R} \$ 724,00$ 
A seguir serão apresentados os principais resultados deste estudo. Primeiramente, será realizada a apresentação dos DSCs gerados a partir das entrevistas com os adolescentes com PC e logo após serão apresentados os DSCs provenientes das entrevistas com suas mães.

\section{Perspectivas Futuras sobre a Participação Social na ótica dos adolescentes}

A partir dos discursos dos adolescentes, é possível identificar positividade em suas perspectivas sobre o futuro, eles também refletem sobre o que será importante para sua qualidade de vida. Os adolescentes enfatizaram a relevância de seguirem uma carreira profissional, de continuarem estudando e estarem perto da família e de amigos.

"Eu, na verdade, penso que vai ser um futuro bem-sucedido, eu sonho em me formar, que vou estar na faculdade, vou ter uma profissão, nunca parar de estudar, então eu vou ter que batalhar muito".

"Para mim (qualidade de vida) é tudo, acho que é ter bom conhecimento pra ter emprego porque hoje em dia se você não é bem qualificado você não tem emprego, então um bom trabalho, dinheiro, felicidade, bons amigos $e$ familiares, gente confiável em volta da gente".

"Não sei, acho que fica meio dificil de te responder".

Ao serem questionados sobre seus sonhos para o futuro, os adolescentes expressaram diferentes desejos e relataram sobre as estratégias consideradas para alcançar seus sonhos, como revelam os discursos a seguir:

"Eu sonho em me formar, fazer uma formatura e depois fazer um curso, até hoje eu não desisti desse sonho”.

"Eu sonho em viajar para fora do Brasil".

"Eu penso em correr atrás, tenho que seguir em frente, trabalhar e estudar bastante, muito mesmo, né? Ai eu consigo".

\section{Perspectivas Futuras sobre a Participação Social na ótica das mães dos adolescentes com PC}

No intuito de se ter uma visão mais ampla sobre as perspectivas sobre o futuro e a participação social dos adolescentes com PC, foi solicitado às suas mães que pudessem compartilhar sua opinião. Assim diferentes aspectos emergiram e dentre eles as expectativas futuras relacionadas à criação de seus filhos adolescentes.

Nesse sentido, as mães demonstraram preocupações com o surgimento de questões inerentes a adolescência, como o envolvimento com pares, namoros, questionamento das práticas parentais e autoafirmação. Além disso, as mães apontaram a necessidade de acompanhar o amadurecimento de seus filhos e reforçar o aprendizado de valores morais considerados importantes. A seguir apresentam-se alguns discursos que retratam estas questões:

"Daqui para frente, o necessário para mim é acompanhar a idade dele, conforme ele passar para adulto? E agir conforme a época dele [...] não vou ter mais aquela fase de criança, de bebê, já passou, agora tenho que continuar".

"Agora vem a adolescência e ela está percebendo mais as coisas ao redor dela. O que agora é bem dificil [...], ela está crescendo e se acha muito grande, se acha já capaz de muita coisa. Agora, ela quer imitar as meninas ao redor dela, e eu tenho que pensar que agora tenho uma filha adolescente em casa. Isso de querer sair com as amigas, como eu vou lidar com isso se ela quiser ir, vai ser dificil".

"Ai mais firmeza na nossa atitude[...] Eles têm que entender que quando a gente fala um "não", não é fácil falar, mas é para o bem deles, então é mais complicado, até então você vai dominando, depois eles vão pegando uma idade que eles acham que tudo pode, mas a gente vai vivendo dia após dia e vai tentando amenizar... Eu acho que tem que ter paciência".

Além disso, as mães demonstraram grande preocupação em auxiliar seus filhos a se prepararem para ter uma educação de qualidade e um bom emprego a fim de garantirem independência financeira e um bom futuro para eles. Conforme ilustrado pelos discursos a seguir:

"Agora com relação ao futuro eu acho que até estou ansiosa com isso, eu falo que ela vai ter que cuidar dela mesma[...] Então eu me preocupo com a situação financeira dela [...] a gente brinca com ela e fala [...] estuda bastante porque quando tiver um concurso e você tiver idade você vai fazer [...] eu não imagino que ela vai ser incapacitada, [...] vai viver de uma aposentadoria porque é deficiente. Nunca pensei nisso! [...] a gente busca que ela tenha independência fisica e que ela tenha independência psicológica [...]". 
"Ah sim, vai ter bastante coisa para eu estar fazendo com ele para estar com uma educação melhor, principalmente [...] para ele não ficar nessas "vidinhas" que tem muitos por aí então a preocupação vai ser maior".

\section{DISCUSSÃO}

Através dos discursos dos adolescentes pode-se compreender que suas aspirações e anseios para a vida futura refletem características típicas da própria adolescência. Eles demonstraram que se planejam em relação ao futuro e a vida adulta, que estabelecem metas, tecem sonhos e expectativas.

Nessa direção, a literatura aponta que a transição da adolescência para a vida adulta pode ser considerada como um período crítico do desenvolvimento, já que os adolescentes precisam tomar várias decisões que refletem sua autonomia em diversas áreas da vida como: trabalho, continuação dos estudos, lazer, cuidados com a saúde, interação social e a participação na comunidade ${ }^{19}$. Assim, os resultados do presente estudo reforçam esta compreensão, na medida em que a preocupação com estes fatores transparece nos discursos dos participantes da pesquisa.

Além disso, importa destacar que apesar de ser importante conhecer o que adolescentes com deficiência física desejam para suas vidas também é fundamental compreender sobre os fatores que podem ajudar ou dificultar essa trajetória. Sem essa compreensão os profissionais que trabalham com esta população podem fazer suposições errôneas sobre o que é importante para estes adolescentes e sobre os fatores que podem ajuda-los no caminho para a vida adulta ${ }^{20}$.

King et al. ${ }^{20}$, em investigação realizada no Canadá sobre os desejos e as metas de vida de adolescentes com PC, entrevistaram 10 adolescentes com idade entre 18 e 20 anos, na fase de transição entre o ensino médio e o curso superior ou entrando no mercado de trabalho. Neste estudo foi questionado aos participantes sobre o que significava ter sucesso na vida, o que eles pensavam que seriam no futuro e os fatores que os ajudavam ou impediam de serem bemsucedidos. Os resultados indicaram que sucesso significava ser feliz. Além disso, três fatores psicossociais foram relacionados com alcançar sucesso e felicidade na vida: acreditar em si mesmos, perceber que os outros também confiam em seu potencial (capacidades) e serem aceitos pelos outros. Nesse sentido, acredita-se que os resultados do presente artigo somam-se com os achados da pesquisa de King ${ }^{20}$, e ressalta-se que os adolescentes ao falarem sobre suas aspirações para o futuro e os desejos da participação demonstram valorizar elementos semelhantes.
Outros aspectos importantes ressaltados nos discursos dos adolescentes foi a expressão de seus desejos em relação a progressão nos estudos, estabelecimento de uma carreira profissional e estarem próximos de suas famílias e entes queridos.

Estes achados estão em consonância com os resultados do estudo de Cussen et al..$^{13}$ que apontaram três temas relacionados às expectativas de adolescentes com $\mathrm{PC}$ para $\mathrm{o}$ futuro: manter relacionamentos íntimos, escolher a vida futura por si mesmos e o lazer visado para os próximos anos. Dentro desses temas os adolescentes expressaram o desejo de estar perto da família, de amigos e de seus animais de estimação, planejar a carreira profissional, viver em casa e de forma independente, fazer uma viagem em momento futuro. Além disso, Cussen et al. ${ }^{13}$ também ressaltaram a importância de se buscar informações a partir dos próprios adolescentes para que seja possível dar suporte as expectativas dos mesmos, ao que os resultados da presente pesquisa vêm a reforçar.

No presente estudo, um aspecto relevante que merece ser destacado se relaciona às expectativas reveladas pelos adolescentes em relação ao próprio futuro e ao comprometimento da função motora grossa. É relevante destacar que apesar de neste estudo os adolescentes participantes possuírem diferentes níveis de comprometimento motor, os resultados apontaram bastante semelhança nas aspirações para o futuro e na participação social com as de adolescentes com desenvolvimento típico e reforçam os achados de Tan et al. ${ }^{21}$ os quais indicaram que o comprometimento da função motora grossa pode não estar diretamente associada à participação social e às perspectivas futuras.

Enfatiza-se também que os adolescentes indicaram com bastante intensidade o valor e a necessidade dos estudos em suas vidas. Hipotetiza-se que a inserção destes adolescentes desde precocemente na rede regular de ensino possa ser um fator facilitador para a participação social destes adolescentes, e também possa influenciar nos seus projetos de vida futura.

Em relação aos discursos maternos observa-se que as mães demonstraram acompanhar o processo de amadurecimento de seus filhos, e que isso requer mudanças na forma de se relacionarem com os mesmos. As mães destacaram a preocupação de manter a firmeza na educação dos filhos, apesar de compreenderem que nesta fase eles estarão mais propensos a desafiar os limites e a buscarem maior autonomia.

Destaca-se que os discursos maternos não se referiram às questões ligadas somente à deficiência, e sim refletiram preocupações familiares basicamente relacionadas à fase da adolescência ${ }^{22}$. Importa também destacar que as 
mães mostraram-se sensíveis em reconhecer os desafios desta fase e, ao mesmo tempo, reafirmaram a postura de acompanha-los neste novo período, respeitando suas subjetividades e necessidades.

Deste modo, entende-se que os achados do presente estudo corroboram para a compreensão de que para a família é necessário fazer novos arranjos e práticas para assim permitir a independência dos filhos adolescentes e para que eles vivenciem com tranquilidade esse processo de entrar e sair do sistema familiar ${ }^{22,23}$.

Por meio dos discursos maternos apresentados entende-se que os resultados da presente pesquisa são consistentes com os achados de Antle et al. ${ }^{10}$ que apontaram que a maioria dos pais dos adolescentes com PC participantes de sua pesquisa tinham preocupações em relação ao futuro de seus filhos como as oportunidades para continuarem os estudos, estabelecerem uma carreira, serem independentes financeiramente e viverem suas vidas de forma autônoma.

Nesse sentido, compreende-se, que as mães do presente estudo compartilharam preocupações muitos semelhantes, pois elas também assumiram a responsabilidade de auxiliar seus filhos nesta busca de independência e crescimento pessoal.

Ressalta-se que ao falarem sobre o futuro de seus filhos, as mães demonstram reconhecer que elas e suas famílias, como um todo, também sofrerão o processo de transição. Nessa direção a literatura aponta que é preciso compreender que as transições tem impacto em ambos adolescentes e seus familiares ${ }^{24}$. Assim, os pais podem experimentar o estresse advindo do início de um novo estágio na vida de seus filhos e ainda podem experimentar momentos emocionais difíceis ao verem suas expectativas em relação ao futuro de seus filhos serem frustradas ${ }^{24,25}$.

Nesse sentido, ainda que as mães tenham se expressado sobre as estratégias que pretendem utilizar é possível que demandem apoio e espaços para compartilhar, os quais podem ser disponibilizados por técnicos ou serviços que acompanham estas famílias. Destarte, destaca-se a importância de que tais demandas sejam contempladas tanto no âmbito da pesquisa, sendo necessários novos estudos que investiguem como os fatores de suporte podem ser melhor efetivados na transição, quanto na proposição de práticas em serviços que atendem esta população.

\section{CONSIDERAÇÕES FINAIS}

Considera-se que o presente estudo contemplou o objetivo almejado, na medida em que forneceu elementos para a compreensão das perspectivas futuras em relação a participação social de adolescentes com PC sob a ótica tanto dos adolescentes quanto de suas mães.

Destaca-se também que em relação às expectativas destes adolescentes a respeito do seu engajamento ocupacional e participação social no futuro, este estudo fornece elementos que devem contribuir para responder as lacunas da literatura nacional e internacional sobre o tema e permite um avanço na medida em que poucos são os estudos que dão voz aos adolescentes para que possam falar sobre quais são suas aspirações e desejos para a vida futura.

Neste estudo revelou-se que os adolescentes ressaltam a importância de estudarem, estabelecerem uma carreira e terem uma família, como perspectivas para suas vidas no futuro. No entanto, sugere-se estudos futuros que envolvam maior número de participantes e considerem os fatores que possam auxiliar ou dificultar a efetivação de seus projetos futuros e na participação social que possam mediar o suporte social.

Desta forma, compreende-se que a presente pesquisa contribui para o avanço do conhecimento nesta área e dá subsídios para intervenções terapêuticas que visem a ampliação e o fortalecimento da participação social desta população.

Agradecimentos: As autoras agradecem a CAPES pelo financiamento da pesquisa.

Contribuição dos autores no texto: Ambas autoras contribuíram, igualmente, com o processo de redação, discussão dos resultados e fundamentação teórica do estudo.

\section{REFERÊNCIAS}

1. Rosenbaum P, Paneth N, Leviton A, Goldstein M, Bax $\mathrm{M}$, Damiano D, et al. The definition and classification of cerebral palsy. Dev Med Child Neurol. 2007;49:1-44. doi: 10.1111/j.1469-8749.2007.00201.x.
2. Oskoui M, Coutinho F, Dykeman J, Jetté N, Pringsheim T. An update on the prevalence of cerebral palsy: a systematic review and meta-analysis. Dev Med Child Neurol. 2013;55(6):509-19. doi: 10.1111/dmen.12080. 
3. Shikako-Thomas K, Majnemer A, Law M, Lach L. Determinants of participation in leisure activities in children and youth with cerebral palsy: systematic review. Phys Occup Ther Pediatr. 2008;28(2):155-69. doi: 10.1080/01942630802031834.

4. Wagner MNL, Cameto R, Garza N, Levine P. After high school: a first look at the postschool experiences of youth with disabilities. A report from the National Longitudinal Transition Study-2 (NLTS2). Menlo Park, Canadá: SRI International; 2005 .

5. Adamson L. Self-Image, adolescence, and disability. Am J Occup Ther. 2003;57(5):578-81. doi: 10.5014/ajot.57.5.578.

6. Majnemer A, Mazer B. New directions in the outcome evaluation of children with cerebral palsy. Semin Pediatr Neurol. 2004;11(1):11-7. doi: 10.1016/j.spen.2004.01.003.

7. Stewart DA, Lawless JJ, Shimmell LJ, Palisano RJ, Freeman M, Rosenbaum PL, et al. Social Participation of adolescents with cerebral palsy: trade-offs and choices. Phys Occup Ther Pediatr. 2012;32(2):167-79. doi: 10.3109/01942638.2011.631100.

8. BIANCHI-LOPES P. Parentalidade, Participação e suporte social: dando voz aos adolescentes com paralisia cerebral e às suas mães [dissertação]. São Carlos: Universidade Federal de São Carlos; 2016.

9. Stewart D. Transition to adult services for young people with disabilities: current evidence to guide future research. Dev Med Child Neurol. 2009;51:169-73. doi: 10.1111/j.14698749.2009.03419.x.

10. Antle BJ, Mills W, Steele C, Kalnins I, Rossen B. An exploratory study of parents' approaches to health promotion in families of adolescents with physical disabilities. Child Care Health Dev. 2008;34(2):185-93. doi: 10.1111/j.13652214.2007.00782.x.

11. Freeborn D, Knafl K. Growing up with cerebral palsy: perceptions of the influence of family. Child Care Health Dev. 2014;40(5):671-9. doi: 10.1111/cch.12113.

12. Kang LJ, Palisano RJ, King GA, Chiarello LA, Orlin MN, Polansky M. Social participation of youths with cerebral palsy differed based on their self-perceived competence as a friend. Child Care Health Dev. 2012;38(1):117-27. doi: 10.1111/j.1365-2214.2011.01222.x.

13. Cussen A, Howie L, Imms C. Looking to the future: adolescents with cerebral palsy talk about their aspirations a narrative study. Disabil Rehabil. 2012;34(24):2103-10. doi: 10.3109/09638288.2012.672540.

14. Pagano M, Gauvreau K. Princípios de bioestatística. São Paulo: Cengage Learning; 2004.

Recebido em: 20.12.17

Aceito em: 19.03 .18
15. Instituto Brasileiro de Geografia e Estatística - IBGE. Cidades: São Carlos, 2015 [citado 18 jul. 2015]. Disponível em: www.ibge.gov.br.

16. Chagas PSCM, Marisa C, Amaral MF, Vaz DV. Tradução para a língua Portuguesa - Brasil do Gross Motor Function Classification System Family Report Questionnaire - faixas etárias 2-4, 4-6, e 6 a 12 anos. Ontario, Canada: CanChild McMaster University; 2015 [cited 2015 Oct 30]. Available from: https://www.canchild.ca/system/tenon/assets/ attachments/000/000/576/original/GMFCS_Family_ portuguese.pdf.

17. Lefevre F, Lefevre AMC. O discurso do sujeito coletivo. Um novo enfoque em pesquisa qualitativa. Caxias do Sul: Ed. Universidade de Caxias do Sul; 2005.

18. Lefevre F, Lefevre AMC. Pesquisa de representação social: um enfoque qualiquantitativo: a metodologia do discurso de sujeito coletivo. Brasília: Líber livro; 2010.

19. Canha LMN. Transição para a vida adulta no contexto da deficiência. Estudos das variáveis pessoais e sociais associadas a um programa de sucesso e desenvolvimento de um modelo de intervenção inclusivo [tese]. Lisboa: Universidade de Lisboa, Faculdade de Motricidade Humana; 2015. Disponível em: https://www.repository.utl.pt/bitstream/10400.5/11674/1/ Doutoramento_LuciaCanha\%20VF.pdf.

20. King GA, Cathers T, Polgar JM, MacKinnon E, Havens L. Success in Life for older adolescents with cerebral palsy. Qual Health Res. 2000;10(6):734-49. doi: 10.1177/104973200129118796.

21. Tan SS, Wiegerink DJHG, Vos RC, Smits DW, Voorman JM, Twisk JWR, et al. Developmental trajectories of social participation in individuals with cerebral palsy: a multicentre longitudinal study. Dev Med Child Neurol. 2014;56(4):370-7. doi: $10.1111 /$ dmen. 12343 .

22. Souza MTS, Oliveira AL. Fatores de proteção familiares, situações de risco, comportamentos e expectativas de jovens de baixa renda. In: Dell'Aglio DDK, editor. Adolescência e juventude: vulnerabilidade e contextos de proteção. São Paulo: Casa do Psicólogo; 2011. p.47-75.

23. Pratta EMM, Santos MA. Família e adolescência: a influência do contexto familiar no desenvolvimento psicológico de seus membros. Psicol Estud. 2007;12:247-56. http://dx.doi. org/10.1590/S1413-73722007000200005.

24. King GA, Baldwin PJ, Currie M, Evans J. Planning Successful transitions from school to adult roles for youth with disabilities. Children's Health Care. 2005;34(3):193-216. doi: 10.1207/ s15326888chc3403_3.

25. Reiss J, Gibson R. Health care transition: destinations unknown. Pediatrics. 2002;110(Suppl 3):1307. Available from: http://pediatrics.aappublications.org/content/pediatrics/110/ Supplement_3/1307.full.pdf 